Juraci A. Cesar 1,2

Marcelo A. Cavaleti 3

Ricardo S. Holthausen 4

Luis Gustavo S. de Lima 4

\section{Mudanças em indicadores de saúde infantil em um município com agentes comunitários: - caso de Itapirapuã Paulista, Vale do Ribeira, São Paulo, Brasil}

\author{
Changes in child health indicators in \\ a municipality with community health workers: \\ the case of Itapirapuã Paulista, Vale do Ribeira, \\ São Paulo State, Brazil
}

1 Departamento Materno Infantil, Universidade Federal do Rio Grande. Rua Eng. Alfredo Huch 475, Rio Grande, RS 96201-900, Brasil. 2 Takemi Program, Harvard School of Public Health. 677 Huntington Avenue, Boston, MA 02115, U.S.A.

3 Associação Comunitária Alto Vale do Ribeira. Rua Dr. Amilcar Gigante 12 Itapirapuã Paulista, SP 18385-000, Brasil.

4 Departamento de Medicina Social, Universidade Federal de Pelotas. C. P. 464, Pelotas, RS 96001-970, Brasil.

\begin{abstract}
Twelve community health workers (CHWs) were trained to offer basic health care for children under five years of age through household visits in Itapirapuã Paulista, a municipality in Vale do Ribeira, São Paulo State, Brazil. Their tasks included diagnosis, initial treatment, and management of diarrhea and acute respiratory diseases at the household level, growth monitoring, incentives to complete basic immunization, and counseling on infant food supplementation. An average of 409 (396-417) children were visited weekly by CHWs in their homes over the course of three years. In this period, the hospitalization rate dropped drastically, and the use of oral rehydration therapy during bouts of diarrhea increased greatly. However, prevalence of stunting and incidence of low birth weight did not change. Child health indicators (CHI) in this municipality were better than those in a municipality chosen as a control. CHWs may have contributed to improved local CHI, but the results should be interpreted with caution for two reasons: (1) CHI is improving in Brazil countrywide and (2) it is a small program implemented in an area with high morbidity and mortality rates and low availability of health services.

Key words Health Status Indicators; Community Health Agent; Health Services; Child Health
\end{abstract}

Resumo Doze agentes comunitários de saúde (ACS) foram treinados para diagnosticar e iniciar tratamento de diarréia e infeção respiratória no domicílio, monitorar crescimento, incentivar imunização básica e orientar a introdução de alimentos à dieta entre menores de cinco anos no Município de Itapirapuã Paulista, Vale do Ribeira, São Paulo, Brasil. Esses cuidados eram oferecidos através de visitas domiciliares a todas crianças nessa faixa etária, residentes na área de abrangência do ACS. Ao longo de três anos, foram visitadas semanalmente, em média, 409 (396417) crianças. Nesse período, a taxa de hospitalização entre menores de cinco anos foi drasticamente reduzida, e a utilização de soro reidratante oral e a cobertura vacinal básica sensivelmente aumentadas. A prevalência de déficit altura/idade e incidência de baixo peso ao nascer praticamente não se modificaram. Os indicadores de saúde infantil nesse município, foram sistematicamente melhores que os indicadores de um município tomado como controle. É bastante provável que os ACS tenham contribuído para essas melhorias. No entanto, há que considerar o fato de os indicadores de saúde infantil estarem melhorando no Brasil, e que este é um programa de pequena escala implementado em um área com elevados índices de morbimortalidade infantil e baixa oferta de cuidados em saúde.

Palavras-chave Indicadores de Saúde; Agente Comunitário de Saúde; Serviços de Saúde; Saúde Infantil 


\section{Introdução}

Nenhuma estratégia em saúde em grande escala no mundo pobre criou tantas expectativas e sucumbiu tão rapidamente quanto a dos agentes comunitários de saúde (ACS). Tal expectativa foi criada pelo fato de os ACS serem considerados elementos essenciais na universalização de cuidados básicos em saúde, particularmente em áreas rurais (WHO/UNICEF, 1978). O colapso decorreu do excessivo número de atribuições que eles receberam, treinamento inapropriado, suporte deficitário, supervisão inadequada, baixos salários e, sobretudo, pela sua baixa efetividade nos programas nacionais (Berman et al., 1987; Kasshay et al., 1998; Walt, 1990).

Os programas de pequena escala sobreviveram por enfrentarem menores dificuldades logísticas, por desfrutarem de maior comprometimento político local e por restringirem a atuação dos seus ACS a situações específicas, o que lhes conferiu maior efetividade (Berman et al., 1987; Kasshay et al., 1998; Walt, 1990).

Ao tempo em que isso ocorria, final dos anos 80, um diagnóstico de saúde realizado no distrito de Itapirapuã, Vale do Ribeira, São Paulo, revelava altos índices de morbimortalidade infantil e baixa utilização de serviços preventivos de saúde entre menores de cinco anos (Cesar \& Victora, 1990). Esse distrito pertencia a Ribeira, um município com $741 \mathrm{~km}^{2}$ que possuía um único posto de saúde onde um médico, uma enfermeira e cinco auxiliares prestavam atendimento aos seus quase 6 mil habitantes. Essa baixa disponibilidade de serviços de saúde, aliada ao difícil acesso à sede do município, único local onde se prestava atendimento continuado, faziam com que boa parte da população rural ficasse desprovida de cuidados básicos em saúde.

A essa altura, o Programa de Agentes de Saúde do Estado do Ceará se consolidava e, em nível federal, o Ministério da Saúde (MS) implementava, a partir das regiões Norte e Nordeste, o Programa de Agentes Comunitários de Saúde (PACS). Em 1994, somente o PACS, contava com cerca de 30 mil ACS atuando (MS, 2000).

A consolidação do programa cearense, a rápida expansão do PACS e, sobretudo, as dificuldades evidenciadas em Itapirapuã Paulista, fizeram com que um projeto de intervenção a partir de ACS fosse elaborado e ali implementado no início de 1994. Este artigo mostra o que ocorreu com os principais indicadores de saúde infantil nessa localidade, entre abril de 1994 e março de 1997. Além disso, compara estes mesmos indicadores aos de um outro município na mesma região mas que não possuía ACS.

\section{Metodologia}

O Vale do Ribeira, pertencente aos Estados de São Paulo e Paraná, possui cerca de 400 mil habitantes e um dos piores indicadores de saúde e educação do Brasil. De acordo com o Fundo das Nações Unidas para Infância (UNICEF), dentre os 11 municípios com pior índice de desenvolvimento infantil no Estado de São Paulo no ano 2000, oito estão localizados no Alto Vale do Ribeira (UNICEF, 2001). Nessa área encontra-se o então distrito de Itapirapuã, agora o Município de Itapirapuã Paulista desde 1992. Nesse município não há uma única indústria e a agricultura é artesanal, destinada, basicamente, à subsistência. Os bóias-frias proliferam na época de extração de resina. A prefeitura local é o maior empregador, há um funcionário público para cada 25 habitantes.

Em abril de 1994, cinco anos após a realização do diagnóstico de saúde infantil, obteve-se recursos junto à Secretaria de Estado da Saúde e UNICEF para a implementação do programa com ACS no município. Recém-emancipado de Ribeira, Itapirapuã Paulista possuía uma unidade básica de saúde onde um médico e três auxiliares prestavam atendimento para aproximadamente 3 mil habitantes residentes em todo o município.

Inicialmente, dividiu-se o município em 12 áreas. Essa divisão baseou-se, principalmente, no número de habitantes por comunidade e na distância entre um domicílio e outro. Em cada uma dessas áreas, recrutou-se pelo menos um morador. Ao final, 18 candidatos participaram do treinamento que se realizou na sede do município em período integral ao longo de duas semanas. Dividiu-se esse treinamento em uma parte teórica e outra prática. Na parte teórica, repassaram-se conteúdos referentes a imunização básica, identificação e manejo inicial de diarréia e infecções respiratórias, reconhecimento de crianças com grau importante de desidratação, idade adequada à introdução de outros alimentos à dieta infantil e interpretação de curvas de crescimento. A parte prática foi realizada nos domicílios e na unidade básica de saúde. Nessa, ensinou-se como fazer pequenos curativos e mostradas algumas crianças enfermas que lá estavam, especial ênfase foi dada àquelas com pneumonia e desidratação, as principais causas de adoecimento e morte entre menores de cinco anos na localidade. Nos domicílios, demonstrou-se como abordar as famílias e como cadastrar e pesar crianças menores de cinco anos.

Doze ACS, nove mulheres e três homens, foram contratados ao final desse treinamento, 
sendo um por área. Cinco desses ACS haviam completado o segundo grau, seis possuíam o primeiro grau completo e um havia concluído apenas quatro anos de escolaridade. Cada um desses ACS deveria visitar, pelo menos uma vez por semana, toda criança menor de cinco anos residente na sua área de abrangência.

Em um primeiro momento a criança era cadastrada, ocasião em que se coletava informações desde a sua gestação até o momento atual. Dentre essas informações constavam: idade e sexo da criança, realização de pré-natal, idade gestacional em que este foi iniciado, número de consultas realizadas, imunização antitetânica, hábito de fumar materno, tipo e local de parto e peso ao nascer, ocorrência prévia de óbito de irmão com menos de cinco anos, internação hospitalar da criança nos últimos 12 meses, realização de consulta médica nos últimos três meses e aleitamento ao seio materno. Para crianças acompanhadas desde o nascimento, boa parte das perguntas não eram aplicadas porque estas informações já haviam sido coletadas por ocasião do cadastramento. Além dessa ficha de cadastro, cada criança possuía ainda uma ficha de acompanhamento, preenchida semanalmente pelo ACS durante a visita domiciliar. Nessa ficha constava, basicamente, informações sobre a ocorrência, tratamento e manejo de doenças comuns na infância (diarréia, infecção respiratória, doenças de pele, pediculose, escabiose), padrão de amamentação e dieta, imunização, consultas médicas, hospitalizações e, na última semana do mês, verificava-se o peso e a altura da criança.

Tanto a ficha de cadastro quanto a de acompanhamento eram entregues no início de cada mês no escritório do programa, localizado na sede do município. Nesse local, essas fichas eram revisadas e digitadas e, em seguida, esses dados encaminhados à Universidade Federal do Rio Grande (FURG) onde era feita análise de consistência e, a cada três meses, uma listagem de freqüência era produzida. Nessa ocasião, o coordenador do programa (J. A. C.), deslocavase até Itapirapuã Paulista e discutia os resultados obtidos em algumas comunidades. Essa reunião era realizada na sede do município e em pelo menos uma das quatro principais comunidades rurais. Dela participavam moradores locais, principalmente mães, profissionais da unidade de saúde, autoridades locais e, sempre que possível, toda a equipe do programa (secretária, coordenador local e os 12 ACS). Essa visita do coordenador durava uma semana, período em que também, durante dois ou três dias, rediscutia e se necessário, recapacitava os ACS em algumas de suas atribuições. Além dis- so, visitava pelo menos três famílias, escolhidas aletatoriamente durante análise dos dados, para verificar se as visitas haviam sido feitas e se os procedimentos e ou doenças anotados na ficha de acompanhamento tinham, de fato, ocorrido. Em nenhuma ocasião se encontrou diferença entre o anotado na ficha pelo ACS e o que ocorreu segundo as mães, exceto no primeiro trimestre, onde o ACS só considerou como tendo usado soro de reidratação oral (SRO), a criança que usou soro de pacote da Central de Medicamentos (CEME). Também, com finalidade de confirmar a realização da visita, toda vez que o ACS ia a algum domicílio, um dos pais deveria assinar ou deixar impressão digital, na folha de acompanhamento da criança. Dessa forma e com esses controles de qualidade, foram coletados os dados entre abril de 1994 e março de 1997, constantes na Tabela 1.

Com a finalidade de avaliar as mudanças observadas nos indicadores de saúde infantil em Itapirapuã Paulista, dois novos diagnósticos de saúde foram realizados em um mesmo período. Um deles em Itapirapuã Paulista e o outro em Bom Sucesso do Itararé, um município na mesma região. Esses dois municípios foram emancipados na mesma ocasião, 1992. Em 1996, quando esses diagnósticos foram realizados, possuíam a mesma estrutura em saúde (um centro de saúde na sede e um posto na zona rural, um médico em período integral, uma enfermeira e quatro auxiliares), cinco estabelecimentos de ensino (incluindo pré-escolar, fundamental e médio) e recebiam anualmente a mesma quantia do fundo de participação dos municípios: R\$ 919.950,00. Itapirapuã Paulista possuía 3.143 habitantes distribuídos em $406 \mathrm{~km}^{2}$, enquanto Bom Sucesso do Itararé, 2.727 em 133km² (IBGE, 1996).

Dez estudantes concluintes do segundo grau, foram treinados na aplicação de questionários padronizados a todas as mães de crianças menores de cinco anos residentes nesses municípios. As informações coletadas incluíam variáveis demográficas, sócio-econômicas e ambientais da família; assistência à gestação e ao parto; utilização de serviços de saúde (preventivos e curativos); amamentação e dieta e avaliação do estado nutricional da criança baseado nos indicadores peso/altura, peso/idade e altura/idade. $\mathrm{O}$ treinamento durou uma semana e foi realizado pelos coordenadores do estudo (J. A. C. e M. A. C.). Não se realizou estudo piloto por duas razões: (1) todas as famílias com crianças menores de cinco anos, residentes nesses dois municípios seriam incluídos e (2) os questionários a serem aplicados haviam sido amplamente testados e utilizados em in- 
Indicadores básicos de saúde infantil para crianças menores de cinco anos. Itapirapuã Paulista, Vale do Ribeira,

São Paulo, Brasil, abril de 1994 e março de 1997.

\begin{tabular}{|c|c|c|c|c|c|}
\hline \multirow[t]{2}{*}{ Indicadores } & \multirow[t]{2}{*}{$\begin{array}{l}\text { Diagnóstico de } \\
\text { saúde infantil } \\
\text { (julho de 1988)* }\end{array}$} & \multirow[t]{2}{*}{$\begin{array}{l}\text { Diagnóstico de } \\
\text { pré-intervenção } \\
\text { (março de 1994) }\end{array}$} & \multicolumn{3}{|c|}{$\begin{array}{l}\text { Período de intervenção } \\
\text { (número médio de crianças visitadas } \\
\text { semanalmente no período: } 408 \text { ) }\end{array}$} \\
\hline & & & $\begin{array}{l}\text { abril/1994- } \\
\text { março/1995 }\end{array}$ & $\begin{array}{l}\text { abril/1995- } \\
\text { março/1996 }\end{array}$ & $\begin{array}{l}\text { abril/1996- } \\
\text { março/1997 }\end{array}$ \\
\hline $\begin{array}{l}\text { Óbitos entre menores de um ano } \\
\text { nos últimos } 12 \text { meses }\end{array}$ & ND & 6 & 2 & 1 & 2 \\
\hline $\begin{array}{l}\text { Hospitalização entre menores de cinco anos } \\
\text { nos últimos } 12 \text { meses }\end{array}$ & $17,0 \%(29)$ & $13,1 \%(51)$ & $2,0 \%(8)$ & $2,7 \%(11)$ & $2,2 \%(9)$ \\
\hline $\begin{array}{l}\text { Déficit altura/idade ( } \geq-2 \text { desvios padrão } \\
\text { de acordo com o NCHS) }\end{array}$ & $35,1 \%(58)$ & $26,7 \%(104)$ & $24,4 \%(97)$ & $23,0 \%(95)$ & $24,2 \%(101)$ \\
\hline Baixo peso ao nascer & $13,4 \%(22)$ & $12,1 \%(47)$ & $11,1 \%(44)$ & $11,8 \%(49)$ & $11,3 \%(47)$ \\
\hline $\begin{array}{l}\text { Uso de soro reidratante oral durante } \\
\text { os episódios de diarréia }\end{array}$ & $\begin{array}{c}(N=92) \\
21,0 \%(19)\end{array}$ & $\begin{array}{l}(N=172) \\
38,6 \%(66)\end{array}$ & $\begin{array}{c}(N=134) \\
83,3 \%(113)\end{array}$ & $\begin{array}{c}(N=152) \\
96,7 \%(148)\end{array}$ & $\begin{array}{c}(N=138) \\
93,2 \%(127)\end{array}$ \\
\hline Imunização básica completa** & $65,3 \%(111)$ & $72,6 \%(282)$ & $95,8 \%(379)$ & $98,4 \%(406)$ & $95,4 \%(398)$ \\
\hline Total & 171 & 389 & 396 & 413 & 417 \\
\hline
\end{tabular}

ND = não disponível; NCHS = National Center for Health Statistics.

* Incluiu somente crianças residentes no distrito de Itapirapuã, atualmente sede do município.

** Somente para crianças com idade entre 12 e 59 meses, que receberam três doses de vacina contra poliomielite,

difteria, tétano, coqueluche e uma dose contra tuberculose e sarampo.

quéritos realizados em vários estados da $\mathrm{Re}$ gião Nordeste e no extremo sul do Brasil. Esses entrevistadores eram diariamente acompanhados por um dos coordenadores. Além disso, $5 \%$ das entrevistas foram parcialmente repetidas por um dos coordenadores do estudo, a fim de confirmar a sua realização e checar as informações coletadas. A coleta de dados deu-se entre os meses de fevereiro e abril de 1996 nesses dois municípios. Esses questionários eram codificados pelo próprio entrevistador ao final de cada dia de trabalho de campo, e revisados por uma pessoa contratada exclusivamente para este fim. Depois, eram duplamente digitados por pessoas diferentes e comparados, sendo uma destas versões corrigidas. Essas etapas, além da listagem de freqüências das variáveis, foram realizadas utilizando-se o programa Epi Info 6.02 (Dean et al., 1994).

\section{Definição de algumas variáveis citadas neste artigo}

- Soro reidratante oral: somente preparado utilizando colher-medida, soro da CEME ou comprado diretamente nas farmácias.

- Imunização básica completa: considerouse com esquema vacinal básico completo a criança com idade entre 12 e 59 meses, que havia recebido três doses de vacinas contra poliomielite, difteria, tétano, coqueluche, e uma dose contra tuberculose e sarampo. Computaram- se somente doses confirmadas no cartão da criança.

- Baixo peso ao nascer: crianças nascidas com peso menor que $2,5 \mathrm{~kg}$.

- Déficit nutricional: utilizou-se o padrão do National Center for Health Statistics (NCHS), baseado nos indicadores altura/idade, peso/altura e peso/idade (NCHS, 1977). A comparação se deu para crianças com déficit altura/idade igual ou menor que menos dois desvios padrão. - Hospitalização: considerou-se como tendo sido internada a criança que recebeu cuidados médicos em hospital, por um período igual ou superior a 24 horas nos 12 meses anteriores à entrevista.

\section{Resultados}

O número médio de crianças visitadas em uma mesma semana ao longo desses três anos foi de 409, variando de 396 a 417. Considerando que o número médio total de crianças menores de cinco anos, residentes no município nesse período foi de 421, variando de 411 a 429, a perda média máxima de acompanhamento foi da ordem de $3 \%$. Ao longo desses três anos, foram realizadas 66.283 visitas, cerca de 4 a 5 por criança/mês, e o custo mensal por criança foi de $\mathrm{R} \$ 8,17$.

A Tabela 1 mostra dados referentes a três momentos: o diagnóstico de julho de 1988, rea- 
lizado somente na sede do então distrito, o diagnóstico pré-intervenção de março de 1994, e os dados de acompanhamento coletados entre abril de 1994 e março de 1997. Os dados desses dois últimos foram coletados pelos próprios ACS. Nessa tabela, verifica-se acentuada redução no número de óbitos infantis e na taxa de hospitalização, sendo mais acentuado no período de intervenção. A prevalência de déficit altura/idade caiu cerca de $30 \%$ entre 1988 e 1994, mas quase nada se alterou no período de execução do programa. O baixo peso ao nascer praticamente não se modificou. No entanto, o uso de soro reidrantante oral que já havia tido substancial aumento entre 1988 e 1994, praticamente dobrou no período de intervenção, sendo oferecido a 19 de cada 20 crianças com diarréia durante o segundo e terceiro anos de intervenção. A imunização básica completa também aumentou significativamente no período de atuação dos ACS, chegando quase a universalização.

A Tabela 2 compara alguns indicadores de saúde infantil entre o município-intervenção e o tomado como controle. Em termos gerais, Itapirapuã Paulista apresentou melhores indicadores: taxa de hospitalização 3,4 menor, incidência de baixo peso ao nascer $25 \%$ menor e utilização de SRO e cobertura vacinal básica completa cerca de $20 \%$ maior. No entanto, a prevalência de déficit altura/idade foi cerca de $20 \%$ menor em Bom Sucesso do Itararé.

\section{Discussão}

Essa avaliação sugere importante redução no número de óbitos infantis, drástica diminuição na taxa de hospitalização entre menores de cinco anos, discreto impacto sobre a prevalência de déficit altura/idade, ausência de impacto sobre baixo peso ao nascer, substancial aumento na utilização de SRO e na cobertura vacinal básica completa (Tabela 1) e, no geral, melhores indicadores infantis no municípiointervenção em relação ao município-controle (Tabela 2).

Ao se interpretar esses resultados, três importantes limitações devem ser consideradas: (1) o estudo de 1988, incluiu apenas uma área do município onde residiam as crianças de melhor nível sócio-econômico e com razoável acesso a cuidados em saúde. Logo, se todas as crianças do agora município tivessem sido incluídas no estudo, muito provavelmente, a taxa de hospitalização, o déficit altura/idade e a incidência de baixo peso ao nascer teriam sido maiores, enquanto a utilização de SRO nos episó- dios de diarréia e a cobertura vacinal básica completa, menores. Os resultados mostrados podem não refletir, portanto, a real magnitude das mudanças observadas entre 1988 e o diagnóstico pre-intervenção de março de 1994; (2) tanto este diagnóstico de 1994, quanto os dados de acompanhamento das crianças, foram coletados pelos próprios ACS, os intervencionistas. Isso pode superestimar dados favoráveis à intervenção, visto que do sucesso do programa dependia a manutenção do emprego deles. Essa foi uma das razões que nos fez avaliar o programa com entrevistadores independentes entre fevereiro e abril de 1996. Ao se comparar os dados coletados por esses entrevistadores (Tabela 2), com o período correspondente de acompanhamento - abril de 1994 a março de 1996 (Tabela 1) - verifica-se que a taxa de hospitalização foi $18 \%$ menor e a utilização de SRO 12\% maior. Entre os demais indicadores, as diferenças foram muito pequenas. Logo, uma superestimativa de achados favoráveis ao programa parece não se caracterizar; (3) finalmente, trata-se de uma avaliação cujo denominador é bastante pequeno, principalmente para situações de baixa ocorrência como, por exemplo, hospitalizações. Os $18 \%$ de subestimativa referidos significam, apenas, duas internações ao longo de 12 meses. No caso dos óbitos, eventos mais raros ainda, esta limitação se acentua ainda mais. Por essas razões, há que se ter cautela ao interpretar os resultados aqui apresentados.

Em termos globais, a Tabela 1 mostra que houve melhorias significativas na maioria dos indicadores de saúde infantil em Itapirapuã Paulista ao longo desses nove anos. Essas melhorias intensificaram-se no período de execução do programa. No entanto, isso não implica dizer que foram somente os ACS os responsáveis por essas mudanças. Melhorias em indicadores básicos de saúde infantil têm sido retratadas em vários estados brasileiros e cidades de grande e médio porte (Barros et al., 1996; Monteiro \& Freitas, 2000; Svitone et al., 2000). Tais mudanças têm sido atribuídas a melhoria do poder aquisitivo da população, maior e melhor oferta de cuidados em saúde e aumento do fornecimento de água tratada e saneamento básico, principalmente (Monteiro \& Freitas, 2000; Tomasi et al., 1996). Em Itapirapuã Paulista, não foi diferente. A emancipação concedeu ao município, maiores investimentos por parte dos governos Federal e Estadual nas áreas de educação, saúde e transportes, o que acabou gerando vários empregos. Além disso, nesse período, o fornecimento de água tratada, embora presente somente nas áreas de menor 
Tabela 2

Comparação de alguns indicadores básicos de saúde entre crianças menores de cinco anos.

Itapirapuã Paulista e Bom Sucesso do Itararé, Vale do Ribeira, São Paulo, Brasil, 1996.

\begin{tabular}{|c|c|c|}
\hline \multirow[t]{2}{*}{ Indicador } & \multicolumn{2}{|c|}{ Município } \\
\hline & $\begin{array}{l}\text { Itapirapuã } \\
\text { Paulista }\end{array}$ & $\begin{array}{l}\text { Bom Sucesso } \\
\text { do Itararé }\end{array}$ \\
\hline Hospitalização entre menores de cinco anos nos últimos 12 meses $(P=0,000)$ & $3,2 \%(13)$ & $10,8 \%(43)$ \\
\hline \multirow[t]{2}{*}{ Déficit altura/idade (menor ou igual a menos dois desvios-padrão em relação ao NCHS $(P=0,010)$} & $24,3 \%(88)$ & $19,8 \%(75)$ \\
\hline & $(N=393)$ & $(N=379)$ \\
\hline \multirow[t]{2}{*}{ Peso inferior a $2.500 \mathrm{~g}(P=0,273)$} & $10,9 \%(36)$ & $13,7 \%(51)$ \\
\hline & $(N=329)$ & $(N=373)$ \\
\hline \multirow[t]{2}{*}{ Uso de soro reidratante oral durante os episódios de diarréia $(P=0,024)$} & $85,7 \%(54)$ & $69,6 \%(55)$ \\
\hline & $(N=63)$ & $(\mathrm{N}=79)$ \\
\hline $\begin{array}{l}\text { Crianças com idade entre } 12 \text { e } 59 \text { meses, que receberam três doses de vacina contra poliomielite, } \\
\text { difteria, tétano, coqueluche e uma dose contra tuberculose e sarampo }(P=0,000)\end{array}$ & $\begin{array}{l}89,3 \%(283) \\
(\mathrm{N}=317)\end{array}$ & $\begin{array}{l}75,7 \%(228) \\
(\mathrm{N}=301)\end{array}$ \\
\hline Total & $(\mathrm{N}=403)$ & $(\mathrm{N}=398)$ \\
\hline
\end{tabular}

$\mathrm{NCHS}=$ National Center for Health Statistics

risco, aumentou em cerca de $50 \%$, passando de $40 \%$ em 1994 para quase $60 \%$ em 1996 . Também, o esgotamento sanitário que não existia em 1988, e se iniciava em 1994, já cobria, em 1996, um quinto dos domicílios. Assim, Itapirapuã Paulista segue também a mesma tendência secular de melhoria dos indicadores básicos de saúde infantil, claramente identificada nas cidades de Pelotas e São Paulo (Barros et al., 1996; Monteiro \& Freitas, 2000).

A comparação entre os dois municípios do Vale do Ribeira, feita na Tabela 2, mostra que os indicadores de saúde infantil eram sistematicamente melhores em Itapirapuã Paulista que em Bom Sucesso do Itararé. E essa diferença ocorreu, apesar de Bom Sucesso do Itararé apresentar vantagem estatisticamente significativa em relação a Itapirapuã Paulista nas seguintes variáveis: renda familiar, escolaridade materna, imunização completa contra tétano neonatal, nascimento em ambiente hospitalar, distância ao serviço de saúde, presença de eletrodomésticos no domicílio, disponibilidade de sanitário com descarga e fornecimento de água tratada. A magnitude dessas diferenças foi descrita e discutida em maiores detalhes em uma outra publicação (Cesar et al., s.d.).

Considerando-se que Itapirapuã Paulista, apesar das desvantagens acima mencionadas, mostrava, no geral, indicadores de saúde melhores que Bom Sucesso do Itararé, é possível, então, sugerir alguma contribuição dos agentes comunitários de saúde nessas melhorias, embora não seja possível quantificá-la. Essa contribuição pode ter ocorrido em relação à taxa de hospitalização, utilização de SRO e imunizações.
Os diagnósticos de 1988 e de 1994, mostraram que pneumonia e desidratação eram responsáveis por praticamente todas as internações entre menores de cinco anos na localidade. Por essa razão, prioridade foi dada ao diagnóstico precoce e início do tratamento da diarréia e das infecções respiratórias ainda no domicílio. Essas crianças, quando necessário, deveriam ser imediatamente referidas ao centro de saúde local onde, de posse de um encaminhamento feito pelo ACS ou da sua presença, recebiam prioridade de atendimento. É possível que esses procedimentos tenham evitado a ocorrência de algumas internações, o que seria suficiente para explicar a redução observada. O diagnóstico e o manejo adequado dessas doenças pelo ACS, e a parceria com o serviço local de saúde, já se demonstraram efetivas em outros estudos (Ronsmans et al., 1988; Sazawal \& Black, 1992).

Solução de reidratação oral da CEME e colher-medida foram contínua e amplamente disponibilizadas aos ACS. Em presença de alguma criança com diarréia, o ACS deveria ensinar a mãe a prepará-lo, e se necessário, dá-lo à criança. Além disso, os ACS forneciam colhermedida e uma quantidade suficiente de SRO até a próxima visita que, em caso de doença como essa, deveria ocorrer mais de uma vez por semana. A média de 4,5 visitas por crianças/mês sugere que isso pode ter, de fato, ocorrido. O aumento do uso de SRO em programas com ACS já foi, também, identificado no Estado do Ceará (Svitone et al., 2000). Nesse estado, o uso de SRO durante o último episódio de diarréia aumentou 2,3 vezes entre crianças menores de três anos em um período de oito anos, 
enquanto que em Itapirapuã Paulista, aumentou 2,5 vezes entre crianças menores de cinco anos ao longo de dois anos. Além dessa diferença na faixa etária, há que mencionar que o Programa de Agentes de Saúde do Ceará cobria cerca de 850 mil crianças em cerca de $180 \mathrm{mu}$ nicípios, logo as dificuldades logísticas eram muito maiores. De qualquer forma, esses achados reforçam a idéia de que os ACS são capazes de aumentar a utilização de SRO durante os episódios de diarréia nos primeiros anos de vida.

As constantes campanhas de vacinação realizadas no país, associadas ao fato de as equipes responsáveis pela administração das doses deslocarem-se pelos povoados mais populosos do município, podem ter contribuído para o aumento da cobertura vacinal observado em Itapirapuã Paulista. Além disso, cabia ao agente identificar durante as visitas regulares crianças com vacinação atrasada, avisar a mãe e se necessário, no dia da vacinação, providenciar algum meio de transporte, custeado pelo próprio programa, para que a criança fosse levada até o local de vacinação. No Programa de Agentes de Saúde do Ceará, a cobertura vacinal básica completa entre crianças com idade entre 12 e 23 meses, aumentou em $32 \%$ ao longo de oito anos (Svitone et al., 2000), enquanto em Itapirapuã Paulista, o aumento em dois anos, foi de $35 \%$ para crianças com idade entre $12 \mathrm{e}$ 59 meses. Apesar das diferenças já mencionadas entre os dois programas e de o programa cearense não possuir grupo controle, é possí- vel supor que os ACS tenham contribuído para a melhoria desse indicador.

Assim como em outras localidades, os indicadores de saúde em Itapirapuã Paulista vinham melhorando. Algumas dessas melhorias podem ter sido aceleradas pelos ACS pelo fato de eles atuarem em condições específicas, possuírem reduzido número de atribuições, fazerem visitas domiciliares freqüentes, seus encaminhamentos receberem prioridade de atendimento no serviço de saúde e, sobretudo, por atuarem em uma localidade com alto padrão de morbimortalidade infantil, baixa oferta e difícil acesso ao serviço de saúde.

Com a implementação do PACS em 1988, o programa de ACS em Itapirapuã Paulista deixou de existir como inicialmente concebido. $\mathrm{O}$ número de ACS foi reduzido a dois terços, as visitas domiciliares deixaram de ser semanais e passaram a ser mensais, as cinco atribuições que possuíam transformaram-se em cerca de 20 e as reuniões comunitárias deixaram de existir. No início de 2001, extingüiu-se o PACS no município e os ACS passaram a fazer parte do Programa Saúde da Família. No entanto, as atribuições e a forma de atuar dos ACS permaneceram praticamente as mesmas.

Por fim, persiste a necessidade de se medir a efetividade dos ACS. Estudos com maior poder estatístico, presença de grupos-controle adequados e, se possível, utilizando-se de um componente qualitativo, poderiam contribuir para um melhor entendimento desta questão.

\section{Agradecimentos}

À Secretaria de Estado da Saúde e ao Instituto de Saúde de São Paulo, Fundo das Nações Unidas para a Infância e Adolescência (UNICEF), Fundação de Amparo à Pesquisa do Rio Grande do Sul (FAPERGS), Prefeituras Municipais de Itapirapuã Paulista e Bom Sucesso do Itararé e ao Takemi Program, Harvard School of Public Health, Estados Unidos, pelo apoio logístico e financeiro que tornaram possível a execução deste programa; aos agentes comunitários de saúde Antonina Martins, Jacira Santos, Jamilson Almeida, Joice Ferreira, Maria de Lourdes Suntak, Maria Ivonete Sarti, Marlene Andrade, Nilcelene Antunes, Orlando Almeida, Paulo Almeida, Santina Ferreira e Tereza Nunes; à coordenadora e ao secretário local do programa Judite Camargo e Rivail Veloso, respectivamente. 


\section{Referências}

BARROS, F. C.; VICTORA, C. G.; TOMASI, E.; HORTA, B. L.; MENEZES, A. M.; CESAR, J. A.; HALPERN, R.; OLINTO, M. T. A.; POST, C. L.; COSTA, J. D.; MENEZES, F. S.; GARCIA, M. M. \& VAUGHAN, J. P., 1996. Saúde materno-infantil em Pelotas, Rio Grande do Sul, Brasil: Principais conclusões da comparação dos estudos das coortes de 1982 e 1993. Cadernos de Saúde Pública, 12(Sup. 1):87-92.

BERMAN, P. A.; GWATKIN, D. R. \& BURGER, S. E., 1997. Community-based health workers: Head start or false start towards health for all? Social Science and Medicine, 25:443-449.

CESAR, J. A.; CAVALETI, M. A.; HOULTHAUSEN, R. S. \& LIMA, L. G. S., s.d. Reduzindo a utilização de serviços de saúde entre menores de cinco anos em um pequeno município no Vale do Ribeira, SP: A possível contribuição dos agentes comunitários de saúde. [Manuscrito em preparação]

CESAR, J. A. \& VICTORA, C. G., 1990. Avaliando a saúde infantil em uma pequena comunidade: $\mathrm{O}$ estudo de Itapirapuã, Vale do Ribeira, SP. Cadernos de Saúde Pública, 6:455-467.

CUFINO-SVITONE, E.; GARFIELD, R.; VASCONCELOS, M. I. \& ARAUJO-CRAVEIRO, V., 2000. Primary health care lessons from the Northeast of Brazil: The Agentes de Saúde Program. Revista Panamericana de Salud Pública, 7:293-302.

DEAN, A. G.; DEAN, J. A.; COULOMBIER, D.; BRENDEL, K. A.; SMITH, D. C.; BURTON, A. H.; DICKER, R. C.; SULLIVEN, K.; FAGAN, R. F. \& ARNER, T. G., 1994. Epi Info, Version 6: A Word Processing, Database, and Statistics Program for Epidemiology on Microcomputers. Atlanta: Centers for Disease Control and Prevention.

IBGE (Fundação Instituto Brasileiro de Geografia e Estatística), 1996. Cidades: O Brasil, Município a Município. 1 Fevereiro 2001 <www.ibge.gov.br/ cidadesat/ufs/perfil2.php>.
KAHSSAY, H. M.; TAYLOR, M. E. \& BERMAN, P. A., 1998. Community Health Workers: The Way Forward. Public Health in Action 4. Geneva: World Health Organization.

MONTEIRO, C. \& FREITAS, I. C., 2000. Evolução de condicionantes socioeconômicas da saúde na infância na cidade de São Paulo (1984-1996). Revista de Saúde Pública, 34(Sup. 6):8-12.

MS (Ministério da Saúde), 2000. Programa de Agentes Comunitários de Saúde. Brasília: MS.

NCHS (National Center for Health Statistics), 1977. Growth Curves for Children, Birth to 18 Years. Publication (PHS) 78-1650. Hyattsville: U.S. Department of Health, Education and Welfare.

RONSMANS, C.; BENNISH, M. I. \& WIERZBA, T., 1988. Diagnosis and mamagement of disentery by community health workers. Lancet, 2:552-555.

SAZAWAL, S. \& BLACK, R. E., 1992. Meta-analisys of intervention trials on case-management of pneumonia in community settings. Lancet, 340:528-533.

TOMASI, E.; BARROS, F. C. \& VICTORA, C. G., 1996. Situação sócio-econômica e condições de vida: Comparação de duas coortes de base populacional no Sul do Brasil. Cadernos de Saúde Pública, 12 (Sup. 1):15-19.

UNICEF (Fundo das Nações Unidas para a Infância), 2001. Situação Brasileira da Infância. Brasília: UNICEF.

WALT, G., 1990. Community Health Workers in National Programs: Just Another Pair of Hands? Philadelphia: Open University Press.

WHO (World Health Organization)/UNICEF (United Nations Children's Fund). 1978. Primary HealthCare B, Alma-Ata 1978. Report of the International Conference on Primary Care, Health for All Series 1. Geneva: WHO.

Recebido em 10 de abril de 2001

Versão final reapresentada em 14 de novembro de 2001 Aprovado em 10 de maio de 2002 